\title{
EVALUACIÓN DE UMBRALES MÍNIMOS DE CELULARIDAD EN UNIDADES DE SANGRE DE CORDON UMBILICAL EN EL INSTITUTO NACIONAL MATERNO PERINATAL DEL PERÚ
}

\author{
Diana Torres ${ }^{1,2, a, c}$, Willy Cerón,1,3,a,c, Belinda Córdova1,a ${ }^{1,}$ Ricardo Rodríguez ${ }^{1, a}$, Efraín Cabrera ${ }^{1,2, a}$, Raúl Alegría ${ }^{1, b}$, \\ José M. Cóndor ${ }^{1, a, d}$
}

\begin{abstract}
RESUMEN
Con el objetivo de evaluar criterios mínimos de celularidad de las unidades de sangre de cordón umbilical (USCU) según los estándares NETCORD en el Instituto Nacional Materno Perinatal de Lima, Perú, se realizó un estudio transversal que incluyó 100 USCU; se determinó el volumen, el recuento de células nucleadas totales (CNT) por hematología y el número de células CD34+ totales, así como también la viabilidad celular, por citometría de flujo. Se encontró que el $56 \%$ de las USCU no cumplen los umbrales mínimos de celularidad para ser criopreservadas en un banco de sangre de cordón umbilical. Se encontró, además, que las USCU de recién nacidos de mayor peso y de sexo femenino presentan mayor volumen y recuentos de células. En conclusión, es necesario considerar estas variables para optimizar la colecta de las USCU y obtener mayores recuentos de células que permita almacenar unidades de alta calidad en un futuro banco de sangre de cordón umbilical en Perú.
\end{abstract}

Palabras clave: Bancos de sangre; cordón umbilical; sangre fetal; antígenos CD34; trasplante de células madre de sangre del cordón umbilical; peso al nacer; criopreservación; Perú. (fuente: DeCS BIREME).

\section{EVALUATION OF MINIMUM THRESHOLDS OF CELLULARITY IN UNITS OF UMBILICAL CORD BLOOD AT INSTITUTO NACIONAL MATERNO PERINATAL IN PERU}

\begin{abstract}
A cross-sectional study that included 100 units of umbilical cord blood (UCB) was conducted to evaluate the minimum criteria of cellularity in UCB units, according to NetCord standards at Instituto Nacional Materno Perinatal in Lima, Peru. The volume, total count of nucleated cells by hematological tests and total number of CD34+ as well as cell viability by flow cytometry were determined. The study revealed that $56 \%$ of UCB units do not fulfill the minimum criteria of cellularity to be cryopreserved in an umbilical cord blood bank. Furthermore, the UCB units of newborns who weighed more and were female had a higher volume and cell count. In conclusion, these variables must undoubtedly be considered to optimize the collection of UCB units and obtain greater cell counts that enable the storage of high-quality units in a future umbilical cord blood bank in Peru.
\end{abstract}

Key words: Blood banks; umbilical cord; fetal blood; antigens, CD34; cord blood stem cell transplantation; birth weight; cryopreservation; Peru. (source: MeSH NLM).

\section{INTRODUCCIÓN}

Tanto la placenta humana como el cordón umbilical, fueron considerados por muchos años un material de desecho posparto; sin embargo, a partir de las investigaciones realizadas en los años sesenta, las células progenitoras hematopoyéticas $(\mathrm{CPH})$ provenientes de las unidades de sangre de cordón umbilical (USCU) se utilizan en la terapia de diversas patologías hematológicas, así como en enfermedades quimio o radiosensibles ${ }^{(1)}$. Durante los últimos años, estas $\mathrm{CPH}$ provenientes de las unidades de sangre de cordón se han aprovechado en terapia celular por las ventajas que presentan sobre las $\mathrm{CPH}$ de medula ósea y sangre periférica. Sin embargo, hay una limitante: el bajo volumen que se obtiene resulta en una baja cantidad de células CD34+, restringiendo su uso casi exclusivamente a pacientes pediátricos o aquellos con peso corporal menor a $35 \mathrm{~kg}$. Aunque se han desarrollado técnicas para superar esta limitante, como el uso de dos unidades de $\mathrm{CPH}$ de sangre de cordón (proveniente de dos cordones umbilicales) ${ }^{(2,3)}$ y la expansión celular ex vivo, sin embargo, estas alternativas se encuentran en investigación ${ }^{(4,5)}$.

Los bancos de sangre de cordón umbilical (BSCU), son instituciones responsables de la colecta, procesamiento

\footnotetext{
Escuela de Tecnología Médica, Universidad Nacional Mayor de San Marcos. Lima, Perú.

Instituto de Hemaféresis y Terapia Celular "IHEMATEC”. Lima, Perú.

Universidad Peruana Cayetano Heredia. Lima, Perú.

Tecnólogo médico; ${ }^{\mathrm{b}}$ médico; ${ }^{\mathrm{c}}$ magíster en Inmunología; ${ }^{\mathrm{d}}$ doctor en ciencias.

Recibido: 30/07/2015 Aprobado: 10/08/2016
}

Citar como: Torres D, Cerón W, Córdova B, Rodríguez R, Cabrera E, Alegría R, Cóndor JM. Evaluación de umbrales mínimos de celularidad en unidades de sangre de cordon umbilical en el Instituto Nacional Materno Perinatal del Perú. Rev Peru Med Exp Salud Publica. 2016;33(4):695-9. doi:10.17843/rpmesp.2016.334.2553 
y criopreservación de estas unidades. NETCORD, organización que agrupa BSCU de donadores no emparentados en todo el mundo ${ }^{(5)}$, fue creada con el propósito de estandarizar y mejorar la calidad de las muestras obtenidas. Para tal fin, esta red internacional ha elaborado estándares de funcionamiento de los BSCU, los cuales se agrupan en el documento International Standars for Cord Blood Collection, Processing, Testing, Banking, Selection and Release (1). Estos parámetros estándar indican las pautas que se deben seguir desde el método de colecta hasta la entrega de las USCU para trasplante. Dentro de estos estándares internacionales para trasplante, se recomienda infundir un mínimo de 2,0 x107 células

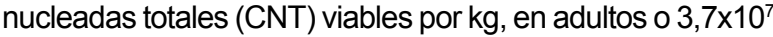
CNT viables/kg en niños ${ }^{(1)}$. Para eso, es necesario obtener un número máximo de células después de la colecta y el proceso de criopreservacion/descongelamiento.

Estudios en BSCU internacionales indican que un alto porcentaje (hasta $80 \%$ ) de USCU son excluidas y no criopreservadas ${ }^{(6,7)}$, la primera causa de exclusión se debe al incumplimiento de los parámetros mínimos de celularidad en relación al volumen y, por tanto, al número de células presentes en la unidad.

El objetivo de este estudio es evaluar el volumen, recuento de CNT, recuento de células CD34+ de las USCU obtenidas en el Instituto Nacional Materno Perinatal (INMP) de Lima y compararlos con los criterios mínimos de celularidad NETCORD para establecer el porcentaje que satisface los criterios de aceptación para ser criopreservadas.

\section{MATERIALES Y MÉTODOS}

Se realizó un estudio transversal entre los meses de marzo a mayo de 2014, fueron colectados USCU de recién nacidos de madres que aceptaron y firmaron el consentimiento informado. Las muestras fueron colectadas en bolsas de sangre simple (Terumo, EE. UU.), con $63 \mathrm{~mL}$ de anticoagulante citrato-fostatodextrosa (CPD), en partos vaginales con la técnica de colecta in útero, realizado en el INMP de Lima. Se colectó la máxima cantidad posible de sangre, el volumen se determinó mediante la diferencia del peso de la unidad y el peso inicial de la bolsa con anticoagulante; además, se realizaron los recuentos de CNT, células CD34+ y viabilidad celular. El procesamiento de las USCU se realizó en los laboratorios del Instituto de Hemaféresis y Terapia Celular (IHEMATEC). El recuento de CNT fue determinado en un autoanalizador hematológico ADVIA 2120 (Siemens, Alemania). El recuento de células CD34+, y el porcentaje de viabilidad celular se midió en el citómetro de flujo FACSCalibur (Becton Dickinson, EE. UU.), según el protocolo ISHAGE.

Se analizaron las variables, sexo y peso del recién nacido, con relación a los parámetros mínimos de celularidad, y se determinó el porcentaje de USCU. Se utilizó estadística descriptiva para caracterizar a la muestra, así como pruebas de normalidad (test de Kolmogorov-Smirnov). Los parámetros de celularidad según el sexo del recién nacido, fue comparada mediante la prueba $t$ de Student, un valor de $p<0,05$ fue considerado significativo. Se utilizó correlación de Pearson, con un nivel de significancia $p<0,01$, para correlacionar el peso de recién nacido con las variables de celularidad. Los datos fueron analizados en el programa estadístico STATA 11.0 .

Toda la información se obtuvo previa firma del consentimiento informado y se manejó de manera estrictamente confidencial y solo por parte de los investigadores. El proyecto fue aprobado por el comité de ética del Instituto Materno Perinatal de Lima: CARTA N097-DG/N309-OEAIDE-INMP-13.

\section{RESULTADOS}

Se recolectaron un total de 103 muestras, dos fueron excluidas por presentar coágulos y una, por problemas técnicos en la colecta. Por lo tanto, 100 muestras fueron analizadas por hematología y citometría de flujo. Del total de recién nacidos, el $52 \%$ fueron del sexo femenino. El promedio de peso de los recién nacidos fue de $3332,6 \pm 352,56 \mathrm{~g}$, con un peso mínimo de $2130 \mathrm{~g}$ y un máximo de $4540 \mathrm{~g}$.

Se determinó el porcentaje de USCU que no cumplían los criterios mínimos para ser criopreservadas; comparando los valores obtenidos en nuestras unidades en relación a volumen y celularidad versus los criterios mínimos de celularidad según los estándares NETCORD (Tabla 1). Se obtuvo que $56 \%$ de las USCU analizadas, no cumplieron los criterios mínimos.

Tabla 1. Distribución de frecuencias en las unidades de sangre de cordón umbilical

\begin{tabular}{lccccc}
\hline & NETCORD & Media & DE & Mínimo & Máximo \\
\hline Volumen $(\mathrm{mL})$ & $40-60$ & 63,3 & 21,2 & 24 & 134 \\
CNT $\times 10^{6} / \mathrm{mL}$ & & 6,0 & 2,0 & 1,3 & 11,9 \\
CNT $\times 10^{8} / \mathrm{mL}$ & $\geq 5,0$ & 7,8 & 3,2 & 1,2 & 16,7 \\
CD34+ viables /uL & & 15,7 & 9,4 & 0,6 & 45,0 \\
CD34+ viables $\times 10^{6}$ & $\geq 1,25$ & 2,0 & 1,3 & 0,1 & 6,1 \\
Viabilidad $(\%)$ & $\geq 85$ & 95,8 & 4,9 & 76,3 & 100 \\
\hline
\end{tabular}

CNT: células nucleadas totales; DE: desviación estándar 
Los parámetros de volumen, el recuento de CNT totales (CNT $\left.\times 10^{8}\right)$, el recuento de células CD34+ viables totales $\left(x 10^{6}\right)$ y el porcentaje de viabilidad celular se muestran en la Tabla 1. Del mismo se presenta la distribución de frecuencias de los parámetros de volumen, recuento de CNT y recuento de células CD34+ totales, según el sexo del recién nacido. Se encontró un mayor promedio en el volumen colectado $(p<0,05)$ y mayor promedio para recuento de CNT x10 $\left.10^{8}<0,05\right)$ en las USCU colectadas de recién nacidos de sexo femenino (Tabla 2 ).

Se obtuvo una correlación positiva, estadísticamente significativa, entre el peso al nacer con los parámetros de volumen $(r=0,3359, p<0,01)$, recuento de CNT $(r=0,2873, p<0,01)$ y células CD34+ vivas totales $(r=0,3599, p<0,01)$.

\section{DISCUSIÓN}

Solamente el $44 \%$ de las USCU alcanzaron los umbrales mínimos de celularidad para ser criopreservadas. Además, las USCU de recién nacidos de mayor peso $\mathrm{y}$ de sexo femenino presentaron mayor volumen $y$ recuentos celulares.

Según NETCORD, los umbrales mínimos de volumen para las USCU son entre 40 y $60 \mathrm{~mL}$, sin embargo, la evidencia que correlaciona volumen con índices de injerto hematopoyético sigue siendo poco clara con respecto a los volúmenes mínimos ${ }^{(8)}$. En relación al promedio de volumen de las USCU se obtuvo un promedio de $63,32 \mathrm{~mL}$ con un rango de $24-134 \mathrm{~mL}$, el cual fue inferior al promedio de volumen reportado por Jaime-Pérez et al. (2011), en un estudio realizado en México, donde obtuvieron un volumen medio de 106,3 $\mathrm{mL}$ con un rango de 48,0-213,2 $\mathrm{mL}^{(9)}$.

Así también al estudio realizado por López et al. (2008), en Guatemala, quienes reportaron volúmenes de entre 41,35$158,24 \mathrm{~mL}{ }^{(10)}$. En contraste, Mahantappa et al. (2008), en un estudio realizado en la India, obtuvieron volúmenes entre $10 \mathrm{~mL}$ y $125 \mathrm{~mL}{ }^{(11)}$. Evidenciando así una variabilidad en el volumen de la colecta de las USCU.

El recuento de células nucleadas totales es otro parámetro comúnmente reportado en adición al volumen de las USCU y el más importante a tomar en cuenta cuando se trata de trasplante de $\mathrm{CPH}$ de sangre de cordón umbilical ${ }^{(8)}$. Los estudios reportan una media de $13,0 \times 10^{8}$ con un rango de 7,0 a $29,0 \times 10^{8} \mathrm{CNT}{ }^{(12,13)}$. Un estudio realizado por Liu et al. (2012) en China, donde analizaron solo las unidades con más de $60 \mathrm{~mL}$ de volumen, reportaron una media de 13,32 $\pm 3,63 \times 10^{8}$ CNT ${ }^{(14)}$. En nuestro estudio obtuvimos un promedio de $7,77 \times 10^{8} \mathrm{CNT}$, que es menor en comparación con estos estudios.

En el contexto de trasplante de USCU, el antígeno de superficie CD34 ha sido asociado a tasas de injerto. Por tanto, se ha recomendado tener un valor umbral mínimo de $1,25 \times 10^{6}$ células $\mathrm{CD}^{4} 4^{+}$viables por USCU; sin embargo, la validación científica de este valor sigue siendo discutible (1). Mediante el protocolo ISHAGE, Morales et al. (2011) reportaron un promedio de células $\mathrm{CD} 4^{+}$de $5,04 \times 10^{6}$ con un rango de $1,6-23,2 \times 10^{6}{ }^{(12)} \mathrm{y}$ Volpe et al. (2011) obtuvieron una media de $3,1 \times 10^{6(12)}$. No obstante, nuestras unidades presentaron una media de 2,0 $\times 10^{6}$, con un rango de 0,1-6,1 $\times 10^{6}$ células CD34 ${ }^{+}$ viables totales, reforzando la idea que obtuvimos una baja celularidad.

Según Netcord-Fact ${ }^{(1)}$. En el presente estudio, de las 100 unidades analizadas, solo el $44 \%$ USCU cumplió con los parámetros de volumen, CNT, $\mathrm{CD} 34^{+}$y viabilidad. Por tanto, se tendría que descartar antes del procesamiento y criopreservación el $56 \%$ de las USCU, por baja celularidad. El volumen y los recuentos de células obtenidos están influenciados por diversos factores obstétricos, uno de ellos es el sexo del recién nacido, donde los estudios concuerdan con el nuestro en que las USCU de bebés de sexo femenino tiene mayores recuentos de células. Otro factor importante asociado a estos parámetros es el peso del recién nacido, el cual en nuestro estudio mostró una correlación positiva baja pero estadísticamente significativa ${ }^{(15-18)}$.

Asimismo, un factor determinante para obtener mayor volumen de colecta y, por lo tanto, mayores recuentos celulares, es el tiempo de clampaje del cordón umbilical, el protocolo de tiempo de clampaje de cordón umbilical

Tabla 2. Distribución según el sexo del recién nacido para volumen, recuento de células nucleadas totales y recuento de células CD34+ totales

\begin{tabular}{lccccccccc}
\hline & \multicolumn{3}{c}{ Femenino $(\mathbf{N}=52)$} & \multicolumn{3}{c}{ Masculino $(\mathbf{N}=\mathbf{4 8})$} \\
\cline { 2 - 10 } & Media & DE & Min & Max & Media & DE & Min & Max & Valor $\boldsymbol{p}$ \\
\hline Volumen $(\mathrm{mL})$ & 69,96 & 21,6 & 28 & 134 & 56,12 & 18,4 & 24 & 111 & $\mathrm{p}<0,05$ \\
Células nucleadas totales $\times 10^{8}$ & 8,59 & 3,3 & 1,2 & 16,7 & 6,9 & 2,9 & 2,5 & 13,2 & $\mathrm{p}<0,05$ \\
Células $\mathrm{CD} 34+$ totales $\times 10^{6}$ & 2,15 & 1,2 & 0,3 & 5,9 & 1,84 & 1,3 & 0,1 & 6,1 & $\mathrm{p}=0,06$ \\
\hline
\end{tabular}

DE: Desviación estandar; Min: minimo; Max: máximo; NS: No significativo 
que maneja el personal del Instituto Materno Perinatal de Lima es de entre 1 a 3 min (clampaje tardío), después del nacimiento del bebé ${ }^{(20,21)}$. Pues entre los primeros 2 y 3 min sin clampar el cordón umbilical existe una trasfusión de la sangre placentaria al recién nacido de $35-40 \mathrm{~mL}$ por $\mathrm{kg}$ de peso ${ }^{(21)}$. Esto también podría explicar los recuentos obtenidos en la población del INMPL, más bajos que los reportados en varios estudios realizados en poblaciones extranjeras. Por otro lado, obtuvimos un volumen promedio mayor al sugerido por NETCORD, en un gran porcentaje de USCU el número células fue bajo.

En relación a las limitantes, debe considerarse que en este estudio los recuentos de células CD34+ fueron realizados previo al procesamiento de la unidad, lo que podría indicar que los recuentos celulares podrían disminuir hasta $10 \%$ en un análisis posprocesamiento, tomando en cuenta que la recuperación celular deberá ser mayor al $90 \%{ }^{(2)}$. Existen otros factores que pueden ser analizados en futuros estudios en nuestra población, como el número de embarazos y abortos previos, semana de gestación, peso de la placenta, longitud del cordón umbilical, entre otros, que también podrían influir en las USCU. Por otro lado, el costo del procesamiento y almacenamiento de las USCU es una fuerte limitante para el establecimiento de BSCU en países en vías de desarrollo.
En conclusión, el estudio muestra que hay un alto porcentaje de USCU de nuestra población que no alcanzan los criterios mínimos para ser criopreservadas. Consideramos que hay diversos factores que puedan influir en los recuentos celulares de esas unidades, es así que sugerimos optimizar las colectas para tener un buen almacenamiento y una buena recuperación celular.

\begin{abstract}
Agradecimientos: a la Dra. Rina Zumaeta Beramendi, jefa del Departamento de Patología Clínica, obstetrices, médicos y residentes del Centro Obstétrico del Instituto Materno Perinatal de Lima por su colaboración para la realización de este estudio. A la empresa Becton Dickinson BD y Representaciones Médicas del Perú SRL.

Contribuciones de autoría: DT, WC, BC y RR han participado en la concepción y diseño del artículo; DT y EC participaron en la recolección y obtención de los datos; DT, WC y BC realizaron el análisis e interpretación de datos y brindaron asesoría estadística. Todos los autores participaron en la redacción, revisión crítica del artículo y aprobaron la versión final del artículo.
\end{abstract}

Fuente de financiamiento: Fundación Instituto Hipólito Unanue.

Conflictos de interés: los autores declaran no tener conflictos de interés en la publicación de este artículo.

\section{REFERENCIAS BIBLIOGRÁFICAS}

1. Netcord-Fact. International standards for cord blood collection, banking, and release for administration accreditation manual.5ta edición. 2013

2. Santa Rita M, García G, Cuan J. Donación de sangre de cordón umbilical. Experiencia en Médica Sur. Revista de investigación médica sur. 2009:16(2):50-54.

3. Lamburini A, Malerba C, Picardi A, Amadori S, Calugi A. Placental/ umbilical cord blood: experience of St. Eugenio Hospital collection center. Transplantation Proceedings 2005;37(6):2670-2.

4. Ballen K, Barker J, Stewart S, Greene $\mathrm{M}$, Lane T. Collection and preservation of cord blood for personal use. Biology of blood and Marrow Transplantation 2008;14(3):365-363.

5. Ávila L, Martínez C, Avila J, Becerra A, Jaimes J, Gómez J: Caracterización de los procesos de obtención, transporte, procesamiento y criopreservación de las muestras de cordón umbilical, obtenidas de enero de 2006 a febrero de 2008 en el banco de células Stem de Colombia. Nova - Publicación científica en ciencias biomédicas.
2009:60-65

6. Calderón-Garcidueña E. Evaluación del programa nacional de sangre placentaria CordMX. Centro Nacional de transfusión sanguínea, Banco de Sangre de Cordón Umbilical. Rev Med Inst Mex Seguro Soc 2005; 43 (Supl 1): 127-129.

7. Lamburini A, Malerba C, Picardi A, Amadori S, Calugi A. Placental/ umbilical cord blood: experience of St. Eugenio Hospital collection center. Transplantation Proceedings 2005; 37(6):2670-2672.

8. Allan D, Petraszko T. A review of factors influencing the Banking of collected umbilical Cord blood units. Stem Cell International.2013: 2013:463031

9. Jaime J, Monreal R, Rodríguez L, Mancías C, Herrera J, Gómez D. Evaluation of volume and total nucleated cell count as cord blood selection parameters. Am J Clin Pathol. 2011; 136(5):721-6.

10. Lopez I.Recuento de células progenitoras hematopoyéticas de sangre de cordón umbilical de madres sanas. Universidad San Carlos de
Guatemala.[Tesis de titulación];2008.

11. Mahantappa A, Banik A, Shivshankar J, Rao A: The stem cell in the umbilical Cord Blood is not related to volume and nucleated cell count. Indian Journal of Biotechnology. 2008;7:547-549

12. Morales VH, Milone J, Etchegoyen O, Bordone J, Uranga A. Banco de células progenitoras hematopoyéticas de cordón umbilical. Medicina (Buenos Aires). 2001; 61: 843-848

13. Solves P, Mirabet V, Blanquer A, Delgado-Rosas F, Planelles D, Andrade $\mathrm{M}$, et al. A new automatic device for routine cord blood banking: critical analysis of different volume reduction methodologies. Cytotherapy. 2009; 11(8):1101-7

14. Liu J, He J, Chen S, Qin F, Wang F, Xu G. Cord Blood Banking and Transplantation in China: A Ten Years Experience of a Single Public Bank. Transfus Med Hemother. 2012; 39(1):23-27

15. Mancinelli F, Tamburini A. Optimizing umbilical cord blood collection: impact of obstetric factors versus quality of cord blood units. Transplant Proc. 2006; 38(4):1174-6. 
16. Wu J, Liao C, Chen J, Xu Z, Gu S, Wu S, et al. Analysis of maternal and neonatal factors associated with hematopoietic reconstruction potential in umbilical cord blood units. Zhongguo Shi Yan Xue Ye Xue Za Zhi. 2010; 18(6):153541

17. Jan R, Wen S, Shyr M, Chiang B. Impact of maternal and neonatal factors on CD34+ cell count, total nucleated cells, and volume of cord blood. Pediatr Transplant. 2008. Institute of Medical Sciences, Tzu-Chi University, Taiwan.

18. Wen S, Zhao W, Lin P, Yang K. Associations among birth weight, placental weight, gestational period and product quality indicators of umbilical cord blood units. Transfus Apher Sci. 2012;12(8):868-73

19. Villamonte W, Jerí M, Lajo L, Monteagudo Y, Diez G. Peso al nacer en recién nacidos a término en diferentes niveles de altura en el Perú. Rev Per Ginecol Obstet. 2011;57(3):145-151.

20. Ocmin A, Margoux T, Macazana B, Rocío F. Relación entre clampaje tardío del cordon umbilical y la concentración de hemoglobina en el recién nacido. [Tesis de titulación].Lima: Facultad de Medicina., Universidad Nacional Mayor de San Marcos; 2002.
21. Camarena P. Tiempo de alumbramiento con drenaje de sangre de cordón umbilical en pacientes anémicas y no anémicas del Instituto Materno Perinatal de Lima. [Tesis de titlulación]. Lima: Facultad de Medicina., Universidad Nacional Mayor de San Marcos; 2002.

Correspondencia: Diana Torres Palomino Dirección: Jr. Huascar 1244. Jesús María, Lima - Perú

Teléfono: (+51 1) 330-8163

Correo electrónico:diana_20_89@hotmail.com

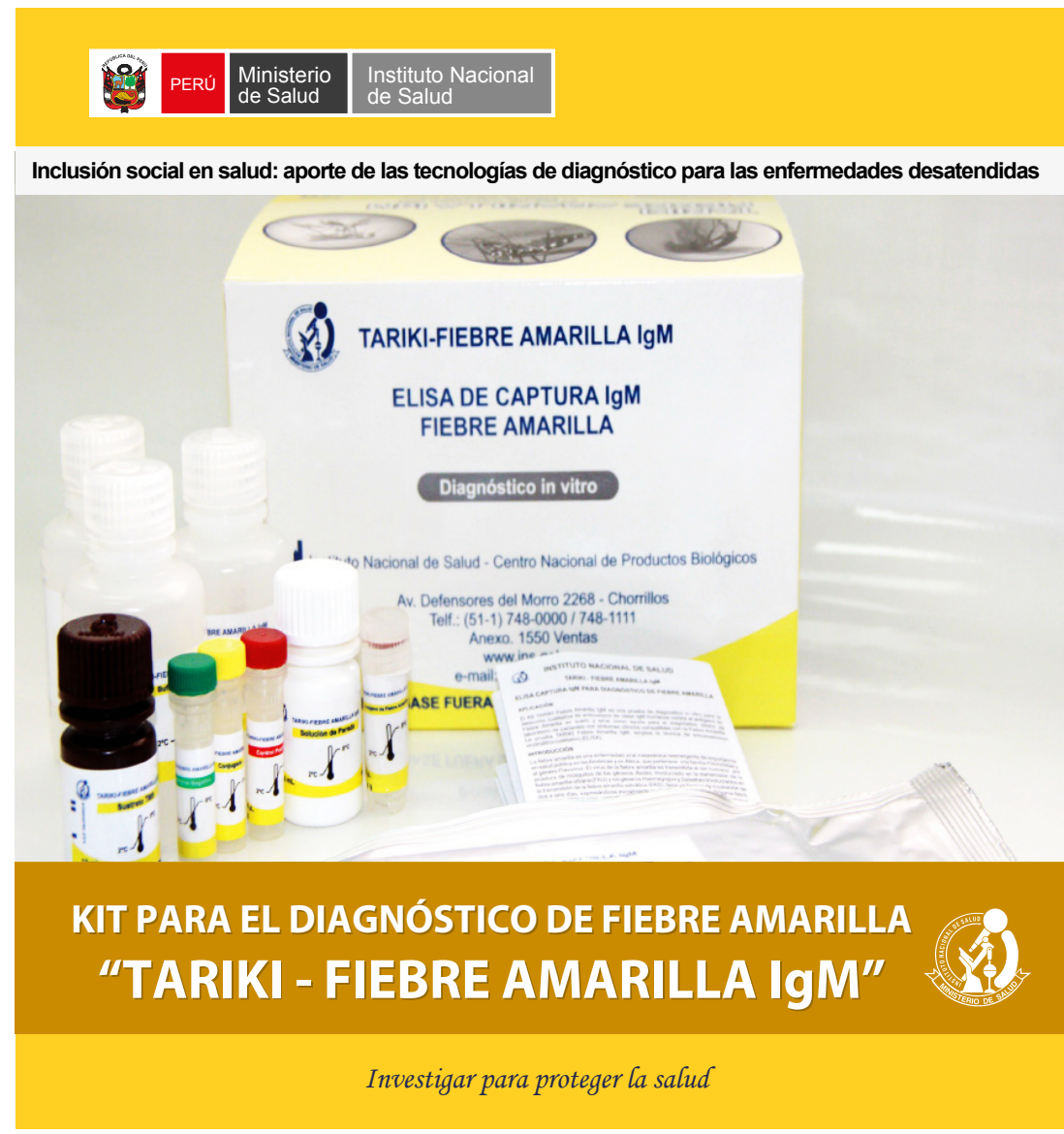

